\title{
Dynamical Phase Trajectories in Baryon and Isospin Density Spaces
}

\author{
M. Colonna ${ }^{\mathrm{a}}$, V. Baran ${ }^{\mathrm{b}}$, M. Di Toro ${ }^{\text {ac }}$ and V. Giordano ${ }^{\mathrm{a}}$ \\ ${ }^{a}$ INFN-LNS, via Santa Sofia 62, I-95123 Catania, Italy \\ ${ }^{\mathrm{b} N I P N E-H H}$, Bucharest and Bucharest University, Romania \\ ${ }^{\mathrm{c} P h y s i c s}$ and Astronomy Dept., University of Catania, Italy
}

We review recent results obtained for charge asymmetric systems at Fermi and intermediate energies, ranging from $30 \mathrm{MeV} / \mathrm{u}$ to $1 \mathrm{GeV} / \mathrm{u}$. Observables sensitive to the isospin dependent part of nuclear interaction are discussed, providing information on the symmetry energy behavior from sub- to supra-saturation densities.

\section{Introduction}

Nuclear reactions give us the opportunity to create transient states of nuclear matter, following several dynamical paths in temperature, baryon and isospin density spaces. By looking at appropriate mechanisms, and related observables, along these trajectories, one can try to map the behavior of the nuclear interaction away from normal conditions. In particular, we want to investigate the energy functional of asymmetric nuclear matter and constrain the term depending on the asymmetry parameter $I=(N-Z) / A$, the so-called symmetry energy, $E_{\text {sym }}$, that is still largely debated nowadays [1,2]. Suitable parametrizations of the symmetry potential can be inserted into existing transport codes (here we will follow the SMF approach [3]), providing predictions for isospin-sensitive observables, that can be confronted to experimental data. We stress that the knowledge of the Equation of State of asymmetric matter (Iso-EOS) has important implications in the context of structure studies and astrophysical problems.

At the Fermi energies, where one essentially explores the low-density zone of the nuclear matter phase diagram, isospin effects can be investigated in reaction mechanisms typical of this energy domain, such as deep-inelastic collisions and multifragmentation. The high density symmetry term can be probed from isospin effects appearing in heavy ion reactions at relativistic energies (few $\mathrm{GeV} / \mathrm{u}$ range). Rather isospin sensitive observables are proposed from nucleon/cluster emissions, collective flows and meson production. A large symmetry repulsion at high baryon density will also lead to an "earlier" hadrondeconfinement transition in n-rich matter.

In the following, we will test an Asysoft parametrization of $E_{\text {sym }}$, with an almost flat behavior below $\rho_{0}$ and even decreasing at supra-saturation, or an Asystiff behavior, with a faster decrease at lower densities and much stiffer above saturation. 


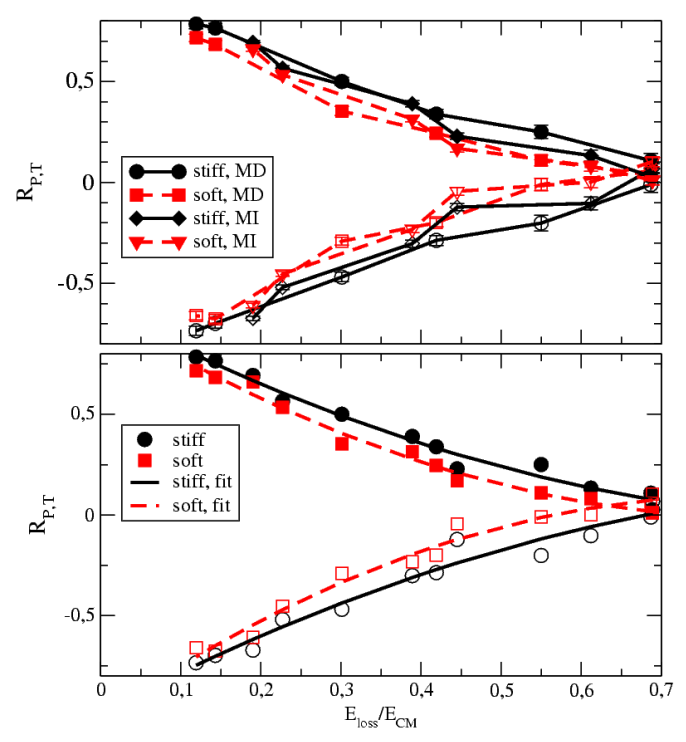

Figure 1. Imbalance ratios as a function of relative energy loss. Upper panel: separately for stiff (solid) and soft (dashed) IsoEOS, and for two parametrizations of the isoscalar part of the interaction: MD (circles and squares) and MI (diamonds and triangles), in the projectile region (full symbols) and the target region (open symbols). Lower panel: quadratic fit to all points for the stiff (solid), resp. soft (dashed) Iso-EOS.

\section{Low density behavior of $E_{\text {sym }}$ : Isospin equilibration}

In this section we focus on the mechanisms connected to isospin transport in binary events at Fermi energies. This process involves nucleon exchange through the low density neck region and hence it is sensitive to the low density behavior of $E_{\text {sym }}$ [4,5,6].

Within a first order approximation of the transport dynamics, the relaxation of a given observable $x$ towards its equilibrium value can be expressed as: $x_{P, T}(t)-x^{e q}=\left(x^{P, T}-\right.$ $\left.x^{e q}\right) e^{-t / \tau}$, where $x^{P, T}$ is the initial $x$ value for the projectile $(\mathrm{P})$ or the target $(\mathrm{T}), x_{e q}=$ $\left(x^{P}+x^{T}\right) / 2$ is the full equilibrium value, $t$ is the elapsed time and $\tau$ is the relaxation time, that depends on the mechanism under study. The degree of isospin equilibration reached in the collision can be inferred by looking at isospin dependent observables in the exit channel, such as the N/Z of PLF and TLF. Using the dissipated kinetic energy as a measure of the contact time $t$, one can finally extract the information on the relaxation time $\tau$, that is related to the symmetry energy. It is rather convenient to construct the socalled imbalance ratio, $R_{P, T}^{x}=\left(x_{P, T}-x^{e q}\right) /\left|x^{P, T}-x^{e q}\right|$ [4]. Within our approximation, it simply reads: $R_{P, T}= \pm e^{-t / \tau}$. The simple arguments developed above are confirmed by full simulations of $(\mathrm{Sn}, \mathrm{Sn})$ collisions at 35 and $50 \mathrm{MeV} / \mathrm{u}[5]$. In figure 10 we report the correlation between $R_{P, T}$ and the total kinetic energy loss, that is used as a selector of the reaction centrality and, hence, of the contact time $t$. On the bottom part of the figure, where all results are collected together, one can see that all the points essentially follow a given line, depending only on the symmetry energy parametrization adopted. A larger equilibration (smaller $R$ ) is observed in the Asysoft case, corresponding to the larger value of $E_{\text {sym }}$. An experimental study of isospin diffusion as a function of the dissipated kinetic energy has been performed recently, by looking at the isotopic content of the light charged particle emission as an indicator of the N/Z of the PLF [7]. This analysis points to a symmetry energy behavior in between the two adopted parametrizations, in agreement with other recent estimates [8]. 


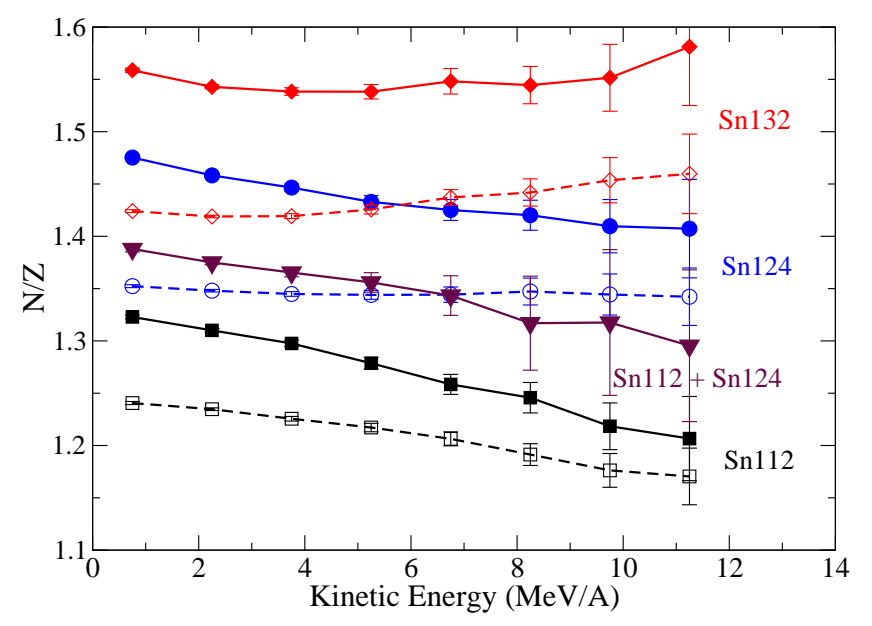

Figure 2. The fragment $\mathrm{N} / \mathrm{Z}$ as a function of the kinetic energy for several $(\mathrm{Sn}, \mathrm{Sn})$ collisions at $\mathrm{E} / \mathrm{A}$ $=50 \mathrm{MeV} / \mathrm{u}$ and $b=2 \mathrm{fm}$. Full lines: Asystiff; Dashed lines: Asysoft.

\section{Isospin distillation in central collisions}

In central collisions at $30-50 \mathrm{MeV} / \mathrm{u}$, where the full disassembly of the system into many fragments is observed, one can study specifically properties of liquid-gas phase transitions occurring in asymmetric matter [1,2,3]. For instance, in neutron-rich matter, phase co-existence leads to a different asymmetry in the liquid and gaseous phase: fragments (liquid) appear more symmetric with respect to the initial matter, while light particles (gas) are more neutron-rich. This sharing of the neutron excess optimizes the energy balance and is ruled by the derivative of the symmetry energy with respect to density. Recently we have proposed to investigate the correlations between the distillation mechanism and the underlying expansion dynamics of the fragmenting system. In fact, in neutron(poor)-rich systems, neutrons(protons) are more repulsed than protons(neutrons), building interesting correlations between the fragment $N / Z$ and kinetic energy, that are sensitive to the symmetry energy parametrization adopted in the calculations, see figure 2. As one can see in the figure, larger (negative) slopes are obtained in the Asystiff case, corresponding to the lower value of the symmetry energy at low density. This appears as a promising experimental observable to be investigated, though fragment secondary effects are expected to reduce the sensitivity to the Iso-EOS [9].

\section{Isospin effects at high baryon density}

The problem of Momentum Dependence in the Isovector channel (Iso-MD) of the nuclear interaction (leading to neutron/proton effective mass splitting) is still very controversial and it would be extremely important to get more definite experimental information [10,11]. Exotic Beams at intermediate energies are of interest in order to have high momentum particles and to test regions of high baryon (isoscalar) and isospin (isovector) density during the reaction dynamics. We present here some results for reactions induced by ${ }^{132} \mathrm{Sn}$ beams on ${ }^{124} \mathrm{Sn}$ targets at $400 \mathrm{MeV} / \mathrm{u}$ [12]. For central collisions in the interacting zone we can reach baryon densities about $1.7-1.8 \rho_{0}$ in a transient time of the order of $15-20 \mathrm{fm} / \mathrm{c}$. In figure 3 we show the $(n / p)$ and ${ }^{3} \mathrm{H} /{ }^{3} \mathrm{He}$ yield ratios at freeze-out, for two choices of mass splitting, vs. transverse momentum $p_{T}$ (upper curves) 

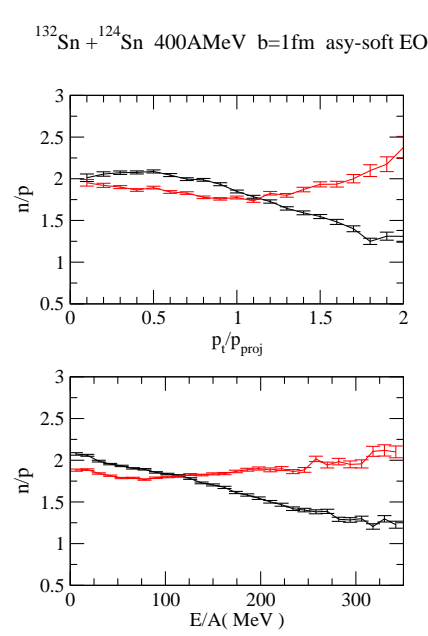
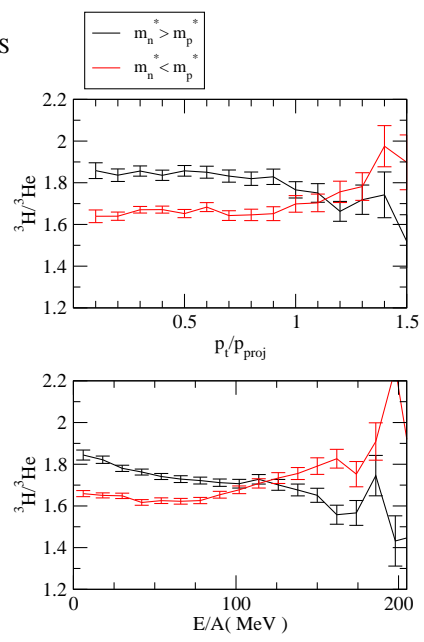

Figure 3. $\quad{ }^{132} \mathrm{Sn}+{ }^{124} \mathrm{Sn}$ at $400 \mathrm{MeV} / \mathrm{u}$, central collisions. Isospin content of nucleon and light ion emissions vs $p_{t}$ (upper) and kinetic energy (lower). Results are shown for the Asysoft interaction

and kinetic energy (lower curves). In this way we can separate particle emission from sources at different densities. We note a clear decreasing trend only in the case $m_{p}^{*}<m_{n}^{*}$, corresponding to a larger proton repulsion. Similar results are obtained for Asysoft or Asystif $f$ parametrizations. Hence these data seem to be suitable to disentangle Iso-MD effects, rather than the stiffness of the symmetry energy. An interesting dependence on the effective mass splitting is observed also for other observables, such as collective flows, that are also sensitive to the Asy-stiffness at high density [12].

\section{Perspectives}

We have reviewed some aspects of the phenomenology associated with nuclear reactions, from which new hints are emerging to constrain the EOS of asymmetric matter. The greatest theoretical uncertainties concerns the high density domain, that has the largest impact on the understanding of the properties of neutron stars. In the near future, thanks to the availability of both stable and rare isotope beams, more selective analyses, also based on new exclusive observables, are expected to provide further stringent constraints.

\section{REFERENCES}

1. V. Baran, M. Colonna, V. Greco, M. Di Toro, Phys. Rep. 410 (2005) 335.

2. B.A. Li, L.W. Chen, C.M. Ko, Phys. Rep. 465 (2008) 113.

3. P. Chomaz, M. Colonna, J. Randrup, Phys. Rep. 389 (2004) 263.

4. M.B. Tsang et al., Phys. Rev. Lett. 92 (2004) 062701.

5. J. Rizzo et al., Nucl. Phys. A806 (2008) 79.

6. S. Wuenschel et al., Phys. Rev. C79 (2009) 061602.

7. E. Galichet et al., Phys. Rev. C79 (2009) 064615.

8. M.B. Tsang et al. Phys. Rev. Lett. 102 (2009) 122701.

9. M. Colonna et al., Phys. Rev. C78 (2008) 064618.

10. B.-A. Li, B. Das Champak, S. Das Gupta, C. Gale, Nucl. Phys. A735 (2004) 563.

11. J.Rizzo, M.Colonna, M.Di Toro Phys. Rev. C72 (2005) 064609.

12. V. Giordano, Master Thesis, Univ. of Catania (2008). 